\title{
COMMON FIXED POINT THEOREMS FOR WEAKLY COMPATIBLE MAPPINGS IN COMPLEX VALUED b-METRIC SPACES
}

\author{
Anil Kumar Dubey ${ }^{1}$, Madhubala Kasar ${ }^{2}$, Ravi Prakash Dubey ${ }^{3}$ \\ ${ }^{1}$ Department of Mathematics, Bhilai Institute of Technology, Bhilai House, Durg, Chhattisgarh 491001, India \\ anilkumardby70@gmail.com \\ ${ }^{2}$ Government Higher Secondary School, Titurdih, Durg, Chhattisgarh 491001, India \\ madhubala.kasar@gmail.com \\ ${ }^{3}$ Department of Mathematics, Dr. C.V. Raman University, Kota, Bilaspur, Chhattisgarh 495113, India \\ ravidubey_1963@yahoo.co.in
}

KeyWords: Complex valued b-metric space, weakly compatible maps, (E.A.) property, (CLRg) property. 2010 MSC : 47H10, 54H25.

\section{Abstract}

In this paper, we prove a common fixed point theorem for weakly compatible mappings in complex valued b-metric space and also improve the condition of contraction of the results of $\mathrm{M}$. Kumar et al.[7]. Further, we prove common fixed point theorems for weakly compatible mappings with (E.A.) property and (CLRg) property.

\section{Introduction and Preliminaries}

In 2011, Azam et al. [2] introduced the notion of complex valued metric spaces and established sufficient conditions for the existence of common fixed points of a pair of mappings satisfying a contractive condition. Complex valued metric space is a generalization of classical metric space. After the establishment of complex valued metric space, many authors have contributed with their works in this space. Some of these results are described in $[7,8,11,13,14]$.

In 2013, Rao et al.[10] introduced the concept of complex valued b-metric space which is a generalization of complex valued metric space. Then, some other authors generalized this concept and proved several common fixed point and fixed point theorems in complex valued b-metric spaces (see [3,4,5,9] and the references contained therein). Aamri and Moutawakil[1] introduced the notion of (E.A.) property. Recently, Verma and Pathak [11] proved common fixed point theorem for two pairs of weakly compatible mappings with (E.A.) property, and a common fixed point theorem using (CLRg) property which was introduced by Sintunavarat and Kumam [12]

In this paper, we prove a common fixed point theorem for weakly compatible mappings satisfying a contractive condition of rational type in the frame work of complex valued b-metric spaces. Further application of perperty (E.A.) and common limit range (CLRg) property are employed.

Let us start by defining some important definitions, basic notations and necessary results from existing literature.

Let $C$ be the set of complex numbers and $z_{1}, z_{2} \in \mathrm{C}$. Define a partial order $\mathbb{\|}$ on $\mathrm{C}$ as follows:

$$
\text { if and only if } \operatorname{Re}\left(z_{1}\right) \leq \operatorname{Re}\left(z_{2}\right), \operatorname{Im}\left(z_{1}\right) \leq \operatorname{Im}\left(z_{2}\right) \text {. }
$$

Thus $z_{1} \mathbb{T} z_{2}$ if one of the following holds:

In particular, we write $z_{1} £ z_{2}$ if $z_{1} \neq z_{2}$ and one of (C1),(C2) and (C3) is satisfied and we write $z_{1} \prec z_{2}$ if only (C3) is satisfied.

Remark 1.1. We note that the following statements hold:

$$
\begin{aligned}
& a, b \in \mathrm{R} \text { and } a \leq b \Rightarrow a z \mathbb{\Phi} b z \forall z \in \mathrm{C} . \\
& 0 \rrbracket z_{1} \mathcal{E}_{z_{2}} \Rightarrow\left|z_{1}\right|<\left|z_{2}\right| . \\
& z_{1} \text { \ } z_{2} \text { and } z_{2} \prec z_{3} \Rightarrow z_{1} \prec z_{3} .
\end{aligned}
$$

Definition 1.2[10] Let $X$ be a nonempty set and let $s \geq 1$ be a given real number. A function $d: X \times X \rightarrow \mathrm{C}$ is called a complex valued b-metric on $X$ if for all $x, y, z \in X$ the following conditions are satisfied:

$$
\begin{aligned}
& 0 \rrbracket d(x, y) \text { and } d(x, y)=0 \text { if and only if } x=y, \\
& d(x, y)=d(y, x)
\end{aligned}
$$




$$
d(x, y) \rrbracket s[d(x, z)+d(z, y)] .
$$

pair $(X, d)$ is called a complex valued b-metric space.

Example 1.3[10] Let $X=[0,1]$. Define a mapping $d: X \times X \rightarrow \mathrm{C}$ by $d(x, y)=|x-y|^{2}+i|x-y|^{2}$, for all $x, y \in X$. Then $(X, d)$ is complex valued b-metric space with $s=2$.

Definition 1.4[10] Let $(X, d)$ be a complex valued b-metric space.

(i) A point $x \in X$ is called interior point of a set $A \subseteq X$ whenever there exists $0 \prec r \in \mathrm{C}$ such that $B(x, r)=\{y \in X: d(x, y) \prec r\} \subseteq A$.

(ii) A point $x \in X$ is called a limit point of a set $A$ whenever for every $0 \prec r \in \mathrm{C}, B(x, r) \cap(A-\{x\}) \neq \phi$.

(iii) A subset $A \subseteq X$ is called an open set whenever each element of $A$ is an interior point of $A$.

(iv) A subset $A \subseteq X$ is called closed set whenever each limit point of $A$ belongs to $A$.

(v) A sub-basis for a Hausdorff topology $\tau$ on $X$ is a family $F=\{B(x, r): x \in X$ and $0 \prec r\}$.

Definition 1.5[10] Let $(X, d)$ be a complex valued b-metric space, $\left\{x_{n}\right\}$ be a sequence in $X$ and $x \in X$.

(i) If for every $c \in \mathrm{C}$, with $0 \prec c$ there is $N \in \mathrm{N}$ such that for all $n>N, d\left(x_{n}, x\right) \prec c$, then $\left\{x_{n}\right\}$ is said to be convergent, $\left\{x_{n}\right\}$ converges to $x$ and $x$ is the limit point of $\left\{x_{n}\right\}$. We denote this by $\lim _{n \rightarrow \infty} x_{n}=x$ or $\left\{x_{n}\right\} \rightarrow x a s n \rightarrow \infty$.

(ii) If for every $c \in \mathrm{C}$, with $0 \prec c$ there is $N \in \mathrm{N}$ such that for all $n>N, d\left(x_{n}, x_{n+m}\right) \prec c$, where $m \in \mathrm{N}$, then $\left\{x_{n}\right\}$ is said to be a Cauchy sequence.

(iii) If every Cauchy sequence in $X$ is convergent, then $(X, d)$ is said to be a complete complex valued b-metric space.

Lemma 1.6[10] Let $(X, d)$ be a complex valued b-metric space and let $\left\{x_{n}\right\}$ be a sequence in $X$. Then $\left\{x_{n}\right\}$ converges to $x$ if and only if $\left|d\left(x_{n}, x\right)\right| \rightarrow 0$ as $n \rightarrow \infty$.

Lemma 1.7[10] Let $(X, d)$ be a complex valued b-metric space and let $\left\{x_{n}\right\}$ be a sequence in $X$. Then $\left\{x_{n}\right\}$ is a Cauchy sequence if and only if $\left|d\left(x_{n}, x_{n+m}\right)\right| \rightarrow 0$ as $n \rightarrow \infty$, where $m \in \mathrm{N}$.

In 1996, Jungck [6] introduced the concept of weakly compatible maps as follows:

Definition 1.8. Two self maps $f$ and $g$ are said to be weakly compatible if they commute at coincidence points. In 2002, Aamri et al. [1] introduced the notion of E.A. property as follows:

Definition 1.9. Two self mappings $f$ and $g$ of a metric space $(X, d)$ are said to satisfy E.A. property if there exists a sequence $\left\{x_{n}\right\}$ in $X$ such that $\lim _{n \rightarrow \infty} f x_{n}=\lim _{n \rightarrow \infty} g x_{n}=t$ for some $t$ in $X$.

In 2011, Sintunavarat et al. [12] introduced the notion of $\left(C L R_{g}\right)$ property as follows:

Definition 1.10. Two self mappings $f$ and $g$ of a metric space $(X, d)$ are said to satisfy $\left(C L R_{g}\right)$ property if there exists a sequence $\left\{x_{n}\right\}$ in $X$ such that $\lim _{n \rightarrow \infty} f x_{n}=\lim _{n \rightarrow \infty} g x_{n}=g x$ for some $x$ in $X$.

2. Main Result In this section, we shall prove our results relaxing the condition of complex valued $b$-metric space being complete.

Theorem 2.1. Let $(X, d)$ be a complex valued b-metric space with the coefficient $s \geq 1$ and let $f, g: X \rightarrow X$. If there exist a mappings $A, B, C, D, E: X \rightarrow(0,1)$ such that for all $x, y \in X$ :

(i) $A(f x) \leq A(g x) ; B(f x) \leq B(g x) ; C(f x) \leq C(g x) ; D(f x) \leq D(g x)$ and $E(f x) \leq E(g x)$, 
(ii) $f X \subseteq g X$,

(iii) $(s A+B+C+D+E) g(x)<1$,

(iv) $d(f x, f y) \rrbracket A(g x) d(g x, g y)+B(g x) \frac{d(g x, f x) d(f y, g y)}{1+d(g x, g y)}$

In $g X$ is complete subspace of $X$, then $f$ and $g$ have a coincidence point. Moreover, if $f$ and $g$ are weakly compatible then $f$ and $g$ have a unique common fixed point.

Proof. Let $x_{0} \in X$, From (ii), we can construct sequences $\left\{x_{n}\right\}$ and $\left\{y_{n}\right\}$ in $X$ by $y_{n}=g x_{n+1}=f x_{n}, n=0,1,2,---$.

From (1), we have

$$
\begin{aligned}
& d\left(y_{n+1}, y_{n}\right)=d\left(f x_{n+1}, f x_{n}\right) \\
& \mathbb{\Upsilon} A\left(g x_{n+1}\right) d\left(g x_{n+1}, g x_{n}\right) \\
& =A\left(f x_{n}\right) d\left(y_{n}, y_{n-1}\right)+B\left(f x_{n}\right) \frac{d\left(y_{n} \cdot y_{n+1}\right) d\left(y_{n} \cdot y_{n-1}\right)}{1+d\left(y_{n}, y_{n-1}\right)} \\
& \text { I } A\left(g x_{n}\right) d\left(y_{n}, y_{n-1}\right)+B\left(g x_{n}\right) \frac{d\left(y_{n} \cdot y_{n+1}\right) d\left(y_{n} \cdot y_{n-1}\right)}{1+d\left(y_{n}, y_{n-1}\right)}
\end{aligned}
$$

Thus, we have

Since $\left|1+d\left(y_{n}, \mathrm{y}_{n-1}\right)\right|>\left|d\left(y_{n}, y_{n-1}\right)\right|$, we have

where $\delta=\frac{A\left(y_{0}\right)+D\left(y_{0}\right)}{1-B\left(y_{0}\right)}<1$.

Now $\left|d\left(y_{n+2}, y_{n+1}\right)\right| \leq \delta\left|d\left(y_{n+1}, y_{n}\right)\right| \leq \delta^{2}\left|d\left(y_{n}, y_{n-1}\right)\right|----$

So for $m>n,\left|d\left(y_{m}, y_{n}\right)\right| \leq s\left|d\left(y_{n}, y_{n+1}\right)\right|+s\left|d\left(y_{n+1}, y_{m}\right)\right|$

Therefore, $\left|d\left(y_{m}, y_{n}\right)\right| \leq \frac{s \delta^{n}}{1-s \delta}\left|d\left(y_{1}, y_{0}\right)\right| \rightarrow 0$ as $n \rightarrow \infty$.

Hence, $\left\{y_{n}\right\}$ is a Cauchy sequence in $g X$. But $g X$ is a complete subspace of $X$, so there is a $u$ in $g X$ such that $y_{n} \rightarrow u$ as $n \rightarrow \infty$. Let $v \in g^{-1} u$. Then $g v=u$. Now, we shall porve that $f v=u$.

$x=v$ and $y=x_{n-1}$ in (1), we get,

Letting $n \rightarrow \infty$, we have

that is $|d(u, f v)| \leq 0$ implies that $f v=u$. Thus, $f v=u=g v$ and hence $v$ is the coincidence point of $f$ and $g$. Now, since $f$ and $g$ are weakly compatible, so $u=f v=g v$ implies that $f u=f g v=g f v=g u$. Now we claim that $g u=u$. Let, if possible, $g u \neq u$.

From (1), we have

that is

Since $|1+d(u, g u)|>|d(u, g u)|$, we have

which implies that $(A+C)(u) \geq 1$, a contradiction.

Hence $g u=u=f u$. Therefore $u$ is the common fixed point of $f$ and $g$. 
For the uniqueness, let $w$ be another common fixed point of $f$ and $g$ such that $w \neq u$.

From (1), we have

that is

Since $|1+d(w, u)|>|d(w, u)|$, we have

$|d(w, u)| \leq(A+C)(w)|d(w, u)|$ implies that $(A+C)(w) \geq 1$, a contradiction. Hence $f$ and $g$ have a unique common fixed point.

Corollary 2.2. Let $(X, d)$ be a complex valued b-metric space with the coefficient $s \geq 1$ and let $f, g: X \rightarrow X$. If there exist a mappings $A, B, C, D: X \rightarrow(0,1)$ such that for all $x, y \in X:$

(i) $A(f x) \leq A(g x) ; B(f x) \leq B(g x) ; C(f x) \leq C(g x) ; D(f x) \leq D(g x)$,

(ii) $f X \subseteq g X$

(iii) $(s A+B+C+D) g(x)<1$,

(iv) $d(f x, f y) \rrbracket A(g x) d(g x, g y)+B(g x) \frac{d(g x, f x) d(f y, g y)}{1+d(g x, g y)}$

In $g X$ is complete subspace of $X$, then $f$ and $g$ have a coincidence point. Moreover, if $f$ and $g$ are weakly compatible then $f$ and $g$ have a unique common fixed point.

Proof. By putting $E=0$ in Theorem 2.1, we get the result of Corollary 2.2.

Corollary 2.3. Let $(X, d)$ be a complex valued b-metric space with the coefficient $s \geq 1$ and let $f, g: X \rightarrow X$. If there exists mapping $A: X \rightarrow(0,1)$ such that for all $x, y \in X$ :

(i) $A(f g) \leq A(g x)$,

(ii) $f X \subseteq g X$,

(iii) $(s A) g(x)<1$,

(iv) $d(f x, f y) \rrbracket A(g x) d(g x, g y)$.

(3) In $g X$ is complete subspace of $X$, then $f$ and $g$ have a coincidence point. Moreover, if $f$ and $g$ are weakly compatible then $f$ and $g$ have a unique common fixed point.

Proof. By putting $B=C=D=E=0$ in Theorem 2.1, we get the result of Corollary 2.3.

Theorem 2.4. Let $(X, d)$ be a complex valued b-metric space with the coefficient $s \geq 1$ and let $f, g: X \rightarrow X$. If there exist a mappings $A, B, C, D, E: X \rightarrow(0,1)$ such that for all $x, y \in X:$

(i) $A(f x) \leq A(g x) ; B(f x) \leq B(g x) ; C(f x) \leq C(g x) ; D(f x) \leq D(g x)$ and $E(f x) \leq E(g x)$,

(ii) $f X \subseteq g X$,

(iii) $(s A+B+C+D+E) g(x)<1$,

(iv) $d(f x, f y) \rrbracket A(g x) d(g x, g y)+B(g x) \frac{d(g x, f x) d(f y, g y)}{1+d(g x, g y)}$

If $f$ and $g$ satisfy $(C L R g)$ property and $f$ and $g$ are weakly compatible then $f$ and $g$ have a unique common fixed point.

Proof. Since $f$ and $g$ satisfy the $(C L R g)$ property, there exists a sequence $\left\{x_{n}\right\}$ in $X$ such that for some $x$ in $X$. 
From (4), we have

$$
d\left(f x, f x_{n}\right) \rrbracket A(g x) d\left(g x, g x_{n}\right)+B(g x) \frac{d(g x, f x) d\left(f x_{n} g x_{n}\right)}{1+d\left(g x \cdot g x_{n}\right)}
$$

Letting $n \rightarrow \infty$, we have

which implies that $|d(f x, g x)| \leq 0$ that is $f x=g x$.

Now, let $u=f x=g x$. Since $f$ and $g$ are weakly compatible mappings, therefore $f g x=g f x$ which implies that $f u=f g x=g f x=g u$.

Now, we claim that $g u=u$. Let, if possible, $g u \neq u$.

From (4), we have

that is

Since $|1+d(u, g u)|>|d(u, g u)|$, then we have

which implies that $(A+C)(u) \geq 1$, a contradiction. Hence $g u=u=f u$.

Therefore, $u$ is the common fixed point of $f$ and $g$.

the uniqueness, let $w$ be another common fixed point of $f$ and $g$ such that $w \neq u$. From (4), we have $d(w, u)=d(f w, f u)$

is $|d(w, u)| \leq A(w)|d(w, u)|+C(w) \frac{|d(w, u) \| d(w, u)|}{|1+d(w, u)|}$.

Since $|1+d(w, u)|>|d(w, u)|$, then we have

implies that $(A+C)(w) \geq 1$, a contradiction. Hence $f$ and $g$ have a unique common fixed point.

Corollary 2.5. Let $f$ and $g$ be self mappings of a complex valued b-metric space $(X, d)$ satisfying all conditions of Theorem 2.4 and the following:

(6)

$$
d(f x, f y) \rrbracket A(g x) d(g x, g y)+C(g x) \frac{d(g x, f y) d(g x, g y)}{1+d(g x, g y)}
$$

for all $x$, $y$ in $X$, where $(s A+C) g(x)<1$. Then $f$ and $g$ have a unique common fixed point.

By putting $B=D=E=0$ in Theorem 2.4, we get the Corollary 2.5.

Corollary 2.6. Let $f$ and $g$ be self mappings of a complex valued b-metric space $(X, d)$ satisfying all conditions of Theorem 2.4 and the following:

$$
d(f x, f y) \rrbracket C(g x) \frac{d(g x, f y) d(g x, g y)}{1+d(g x, g y)}
$$

for all $x$, $y$ in $X$, where $(s C) g(x)<1$. Then $f$ and $g$ have a unique common fixed point.

By putting $A=0$ in Corollary 2.5, we get the Corollary 2.6.

Corollary 2.7. Let $f$ and $g$ be self mappings of a complex valued b-metric space $(X, d)$ satisfying all condition of Theorem 2.4 and the following:

$$
d(f x, f y) \rrbracket A(g x) d(g x, g y)
$$


for all $x$, $y$ in $X$, where $(s A) g(x)<1$. Then $f$ and $g$ have a unique common fixed point.

By putting $C=0$ in Corollary 2.5, we get the Corollary 2.7.

Theorem 2.8. Let $(X, d)$ be a complex valued b-metric space with the coefficient $s \geq 1$ and let $f$ and $g$ be self mappings satisfying all conditions of Theorem 2.4 and the following:

(a) $f$ and $g$ satisfy E.A. Property and $f$ and $g$ are weakly compatible,

(b) $g X$ is a closed subset of $X$.

Then $f$ and $g$ have a unique common fixed.

Since $f$ and $g$ satisfy the E.A. property, there exists a sequence $\left\{x_{n}\right\}$ in $X$ such that for some $x$ in $X$.

(9)

Now, $g X$ is closed subset of $X$, therefore $\lim _{n \rightarrow \infty} g x_{n}=g a$, for some $a \in X$. So from (9), we have $\lim _{n \rightarrow \infty} f x_{n}=g a$. We claim that $f a=g a$.

From (4), we have

$$
d\left(f a, f x_{n}\right) \rrbracket A(g a) d\left(g a, g x_{n}\right)+B(g a) \frac{d(g a, f a) d\left(f x_{n}, g x_{n}\right)}{1+d\left(g a \cdot g x_{n}\right)}
$$

Letting $n \rightarrow \infty$, we have

which implies that $|d(f a, g a)| \leq 0$, that is $f a=g a$.

Now, we show that $f a$ is the common fixed point of $f$ and $g$. Let, if possible $f a \neq f f a$.

Since $f$ and $g$ are weakly compatible, $g f a=f g a$ implies that $f f a=f g a=g f a=g g a$.

From (4), we have

$$
\begin{aligned}
& d(f f a, f a) \mathbb{T}(g f a) d(g f a, g a)+B(g f a) \frac{d(g f a, f f a) d(f a, g a)}{1+d(g f a, g a)} \\
& +C(g f a) \frac{d(g f a, f a) d(g f a, g a)}{1+d(g f a, g a)} \\
& +D(g f a) \frac{d(g a, f a) d(g f a, g a)}{1+d(g f a, g a)} \\
& +E(g f a) \frac{d(g f a, f a) d(f a, g a)}{1+d(g f a, g a)} \\
& =A(f f a) d(f f a, f a)+B(f f a) \frac{d(f f a, f f a) d(f a, g a)}{1+d(f f a, g a)} \\
& +C(f f a) \frac{d(f f a, f a) d(f f a, g a)}{1+d(f f a, g a)} \\
& +E(f f a) \frac{d(f f a, f a) d(f a, g a)}{1+d(f f a, g a)}
\end{aligned}
$$


$=A(f f a) d(f f a, f a)+C(f f a) \frac{d(f f a, f a) d(f f a, f a)}{1+d(f f a, f a)}$

that is,

$|d(f f a, f a)| \leq A(f f a)|d(f f a, f a)|$

$+C(f f a) \frac{|d(f f a, f a) \| d(f f a, f a)|}{|1+d(f f a, f a)|}$.

Since $|1+d(f f a, f a)|>|d(f f a, f a)|$,

we have $|d(f f a, f a)| \leq(A+C)(f f a)|d(f f a, f a)|$, a contradiction, since $(A+C) \geq 1$.

Hence $f f a=f a=g f a$. Thus $f a$ is common fixed point of $f$ and $g$.

Finally, we show that the common fixed point is unique. For this, let $u$ and $v$ be two common fixed point of $f$ and $g$ such that $u \neq v$.

that is

Since $|1+d(u, v)|>|d(u, v)|$,

we have $|d(u, v)| \leq(A+C)(u)|d(u, v)|$, a contradiction, since $(A+C) \geq 1$.

Hence $f$ and $g$ have a unique common fixed point.

Corollary 2.9. Let $(X, d)$ be a complex valued b-metric space with the coefficient $s \geq 1$ and let $f$ and $g$ be self mappings satisfying the following:

(i) $f X \subseteq g X$,

(ii) $f$ and $g$ satisfy E.A. property and $f$ and $g$ are weakly compatible,

(iii) $(s A+C) g(x)<1$,

(iv) $d(f x, f y) \llbracket A(g x) d(g x, g y)+C(g x) \frac{d(g x, f y) d(g x, g y)}{1+d(g x, g y)}$,

for all $x, y$ in $X$.

Then $f$ and $g$ have a unique common fixed point.

Proof. By putting $B=D=E=0$ in Theorem 2.8, we get the Corollary 2.9.

Corollary 2.10. Let $f$ and $g$ be self mappings of a complex valued b-metric space $(X, d)$ satisfying all conditions of Corollary 2.9 and the following:

$$
d(f x, f y) \rrbracket C(g x) \frac{d(g x, f y) d(g x, g y)}{1+d(g x, g y)}
$$

for all $x, y$ in $X$, where $(s C) g(x)<1$.

Then $f$ and $g$ have a unique common fixed point.

Proof. By putting $A=0$ in Corollary 2.9, we get the Corollary 2.10.

Corollary 2.11. Let $f$ and $g$ be self mappings of a complex valued b-metric space $(X, d)$ satisfying all conditions of Corollary 2.9 and the following:

$d(f x, f y) \rrbracket A(g x) d(g x, g y)$

for all $x, y$ in $X$, where $(s A) g(x)<1$. 
Then $f$ and $g$ have a unique common fixed point.

Proof. By putting $C=0$ in Corollary 2.9, we get the Corollary 2.11.

\section{References}

1. M. Aamri and D. El. MoutawaKil, Some new common fixed point theorems under strict contractive conditions, J. Math. Anal. Appl. 27(1), 181-188(2002).

2. A.Azam, B.Fisher and M. Khan, Common fixed point theorems in complex valued metric spaces, Numer. Funct. Anal. Optim. 32(3),243-253(2011).

3. A.K. Dubey, Common fixed point results for contractive mappings in complex valued b-metric spaces, Nonlinear Funct. Anal. and Appl., Vol.20, No.2, 257-268(2015).

4. A.K. Dubey, Rita Shukla, R.P. Dubey, Some Fixed Point Theorems in Complex Valued b-Metric Spaces, Journal of Complex Systems, Vol. 2015, Article ID 832467, 7 Pages, http.//dx.doi.org/10.1155/2015/832467.

5. A.K. Dubey, Rita Shukla, R.P. Dubey, Some common fixed point theorems for contractive mappings in complex valued b-metric spaces, Asian Journal of Mathematics and Applications, Vol. 2015, Article ID ama0266, 13 pages.

6. G. Jungck, Common fixed points for non-continuous non-self mappings on non-metric spaces, Far East J. Math. Sci. 4(2), 199-212(1996).

7. M. Kumar, P. Kumar, S. Kumar, Common fixed point theorems in Complex valued metric spaces, Journal of Analysis \& Number Theory, 2(2), 103-109(2014).

8. S. Kumar, M. Kumar, P. Kumar, S.M. Kang, Common fixed point theorems for weakly compatible mappings in complex valued metric spaces, Int. J. of Pure and Applied Mathematics, 92(3), 403-419(2014).

9. A.A. Mukheimer, Some common fixed point theorems in complex valued b-metric spaces, The Scientific World Journal, Vol.2014(2014), Article ID 587825, 6 pages.

10. K.P.R Rao, P.R Swamy, J.R., Prasad, A common fixed point theorem in complex valued b-metric spaces, Bulletin of Mathematics and Statistics Research, Vol. 1, Issue 1,1-8(2013).

11. R.K. Verma, H.K. Pathak, Common fixed point theorems using property (E.A.) in complex valued metrid spaces, Thai J. of Mathematics, 11(2), 347-355(2013).

12. W. Sintunavarat, P. Kumam, Common fixed point theorems for a pair of weakly compatible mappings in fuzzy metric spaces, Journal of Applied Mathematics, 2011, Article ID 637958, 14 pages.

13. W. Sintunavarat, P. Kumam, Generalized common fixed point theorems in complex valued metric spaces and applications, Journal of Inequalities and Applications, (2012), 2012:84

14. W. Sintunavarat, Y.J. Cho, P. Kumam, Urysohn integral equations approach by common fixed points in complex valued metric spaces, Advan ces in Difference Equations, 2013(2013):49. 